\title{
Teratogenic Potential of Antiepileptic Drugs in the Zebrafish Model
}

\author{
Sung Hak Lee, Jung Won Kang, Tao Lin, Jae Eun Lee, and Dong Il Jin \\ Department of Animal Science \& Biotechnology, Chungnam National University, Daejeon 305-764, Republic of Korea
}

Correspondence should be addressed to Dong Il Jin; dijin@cnu.ac.kr

Received 9 July 2013; Accepted 9 October 2013

Academic Editor: Daiana Avila

Copyright (C) 2013 Sung Hak Lee et al. This is an open access article distributed under the Creative Commons Attribution License, which permits unrestricted use, distribution, and reproduction in any medium, provided the original work is properly cited.

The zebrafish model is an attractive candidate for screening of developmental toxicity during early drug development. Antiepileptic drugs (AEDs) arouse concern for the risk of teratogenicity, but the data are limited. In this study, we evaluated the teratogenic potential of seven AEDs (carbamazepine (CBZ), ethosuximide (ETX), valproic acid (VPN), lamotrigine (LMT), lacosamide (LCM), levetiracetam (LVT), and topiramate (TPM)) in the zebrafish model. Zebrafish embryos were exposed to AEDs from initiation of gastrula (5.25 hours post-fertilization (hpf)) to termination of hatching ( $72 \mathrm{hpf}$ ) which mimic the mammalian teratogenic experimental design. The lethality and teratogenic index (TI) of AEDs were determined and the TI values of each drug were compared with the US FDA human pregnancy categories. Zebrafish model was useful screening model for teratogenic potential of antiepilepsy drugs and was in concordance with in vivo mammalian data and human clinical data.

\section{Introduction}

Antiepileptic drug (AED) exposure in utero has been associated with major congenital malformations (MCMs) and adverse cognitive outcomes in the offspring of women with epilepsy (WWE) [1,2]. The use of older-generation AEDs during pregnancy is known to be associated with a two- to threefold increased risk of birth defects in the offspring and possibly also other adverse outcomes in the exposed infant. Much less has been known about newer-generation AEDs [3].

Preclinical toxicological studies include testing for teratogenicity in at least two different species. However, such animal teratology studies are generally expensive and time consuming [4]. Clinical studies on the teratogenic effects of AEDs have been too small and underpowered to enable researchers to draw significant conclusions [5].

There is a need to develop a teratogenicity assay for its ability to predict the teratogenic potential of drugs. The criteria for a useful teratogenicity screening should include costeffectiveness, adequate throughput, straightforward assay conduct, reproducibility, and concordance with in vivo mammalian data $[4,6]$.

Zebrafish are inexpensive and easy to maintain and breed in large numbers [7, 8]. Furthermore, zebrafish development is similar to that of mammals, and many molecular pathways are evolutionarily conserved between zebrafish and humans [4]. Owing to these advantages, zebrafish embryo is considered a suitable alternative model for traditional in vivo developmental toxicity screening [9].

In 1975, the FDA created guidelines for drug companies to follow in regard to labeling medications about their effects on reproduction and pregnancy. The pregnancy category of a pharmaceutical agent is an assessment of the risk of fetal injury due to the pharmaceutical. The FDA has a categorization of drug risks to the fetus that runs from "Category A" to "Category X" [10].

In this study, we evaluated the teratogenic potential of seven AEDs: carbamazepine (CBZ, FDA drug pregnancy category "D”), ethosuximide (ETX, “C”), valproic acid (VPN, “D”), lamotrigine (LMT, “C”), lacosamide (LCM, “C”), levetiracetam (LVT, "C"), and topiramate (TPM, "D") in the zebrafish model. Zebrafish embryos were exposed to AEDs from initiation of gastrula (5.25 hours post-fertilization (hpf)) to termination of hatching ( $72 \mathrm{hpf}$ ) which mimic the exposure time of mammalian teratogenic experimental design. The lethality and teratogenicity were determined and used to calculate the teratogenic index (TI). To evaluate the concordance of the TI values in zebrafish model with in vivo 
mammalian data and human clinical data, we compared the correlation TI values in zebrafish embryos with FDA categories of seven AEDs.

\section{Materials and Methods}

2.1. Animals. Adult zebrafish (wild-type AB strain) of either sex were obtained from a commercial supplier (OK aquamall, Gyeonggi-Do, Korea). Zebrafish were housed separately by gender under a $14 \mathrm{~h}$ light $/ 10 \mathrm{~h}$ dark cycle and fed live brine shrimp 2-3 times a day. The water temperature was maintained at $28 \pm 1^{\circ} \mathrm{C}$ and $\mathrm{pH}$. The day before spawning, two pairs of adult zebrafish were placed in a breeding tank equipped with a spawning tray. Eggs were collected and placed in Petri dishes filled with egg water $(60 \mu \mathrm{g}$ ocean salt $/ \mathrm{mL}$ ) [8]. Shortly after spawning, eggs were collected from the cage, and fertilized eggs were selected for all experiments. All animal care and use procedures were approved by the Institutional Animal Care and Use Committee of Chungnam National University.

2.2. Test Drugs. Drugs were purchased from Sigma-Aldrich (St. Louis, MO, USA) and Hanchem Co., Ltd. (Daejeon, Korea). Carbamazepine (CBZ, CAS no. 298-46-4, purity $100 \%$ ), ethosuximide (ETX, CAS No. 77-67-8, purity 99.9\%), and valproic acid sodium salt (VPN, CAS no. 1069-655, purity 99.9\%) were purchased from Sigma-Aldrich, and lamotrigine (LMT, CAS no. 84057-84-1, purity > 97\%), lacosamide (LCM, CAS no. 175481-36-4, purity > 97\%), levetiracetam (LVT, CAS no. 102767-28-2, purity > 97\%), and topiramate (TPM, CAS no. 97240-79-4, purity > 97\%) were purchased from Hanchem Co., Ltd.

2.3. Drug Exposure of Zebrafish Embryos. CBZ, LCM, LMT, and TPM were dissolved in DMSO (Sigma-Aldrich, St. Louis, MO, USA) and the remaining drugs dissolved in egg water. Typically, 5 to 6 selected embryos were transferred to 24 multiwell plates (Becton Dickinson, Franklin Lakes, NJ, USA). DMSO $(10 \mu \mathrm{L})$ or egg water solution $(50 \mu \mathrm{L})$ was added to $1 \mathrm{~mL}$ aliquots of egg water. DMSO $(1 \%, \mathrm{v} / \mathrm{v})$ served as the control solution.

Embryos were exposed to test compounds from initiation of gastrula (5.25 hpf) to termination of hatching (72 hpf) [11]. This exposure duration mimics that of rodent embryonic development (implantation to closure of the hard palate) (Figure 1).

2.4. Evaluation of Lethality and Teratogenic Effects. Endpoints were combined and modified based on the procedures of [4, 12,13 . Embryos were examined daily and evaluated at $72 \mathrm{hpf}$. Lethal or teratogenic effects were recorded under an Olympus SZ61 stereomicroscope (Tokyo, Japan). The $8 \mathrm{hpf}$ time point served as a control step to identify unfertilized eggs.

Lethality endpoints (coagulation of the embryo, nondetection of the heartbeat) and teratogenicity endpoints (malformation of the head, tail, or heart, scoliosis, deformity of yolk, and growth retardation) were evaluated under a microscope at $72 \mathrm{hpf}$.

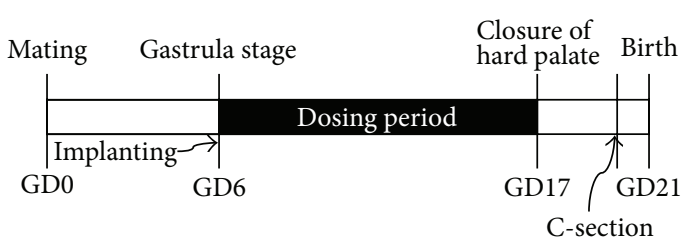

(a)

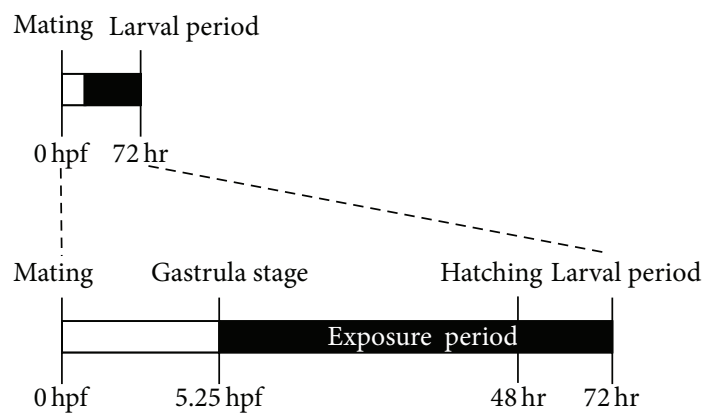

(b)

FIgURE 1: The exposure time of test compounds was modified to concur with those of the rodent teratogenicity study. (a) Typical experimental method for rat. GD: gestation day. (b) Modified method for zebrafish. hpf: hours post-fertilization.

2.5. Calculation of the Teratogenicity Index (TI). In order to characterize the teratogenic potential of a test substance, the teratogenicity index (TI), which was defined as the quotient of $\mathrm{LC}_{50}$ and $\mathrm{EC}_{50}$, was calculated $[12,14]$.

2.6. Statistical Analysis. Egg batches were only used at fertilization rates of $\geq 90 \%$. An assay was considered valid if the controls did not show $>10 \%$ teratogenic plus lethal effects at $72 \mathrm{hpf}$. $\mathrm{LC}_{50}$ and $\mathrm{EC}_{50}$ values were measured with PHARM/PCS (Version 4, Murray Springer-Verlag). The teratogenicity was analyzed using one-way analysis of variance (ANOVA), followed by Dunnett's multiple comparison test (Version 5.0, GraphPad Prism for Windows).

\section{Results}

Zebrafish embryos were exposed to AEDs from initiation of gastrula $(5.25 \mathrm{hpf})$ to termination of hatching (72 hpf). The concentrations of each AED were chosen based on preliminary experiments (data not shown) and their feasible solubility. All controls fulfilled the acceptance criteria, specifically, $\geq 90 \%$ fertilization rate and $\leq 10 \%$ teratogenic effect.

3.1. Lethal and Teratogenic Effects of Antiepileptic Drugs. The lethality and teratogenicity of antiepileptic drugs were determined and calculated as TI at $72 \mathrm{hpf}$. Results are presented in Figure 2. The $\mathrm{LC}_{50}$ and/or $\mathrm{EC}_{50}$ values of LMT and LVT could not be calculated based on the solubility limit. TI values were ranked as follows: $\mathrm{VPN}>\mathrm{TPM}>\mathrm{LCM}>\mathrm{CBM}>$ $\mathrm{LMT}>\mathrm{LVT} \cong \mathrm{ETX}$.

3.2. Malformation Effects of Antiepileptic Drugs. The teratogenicity endpoints (malformation of head, tail, or heart, 


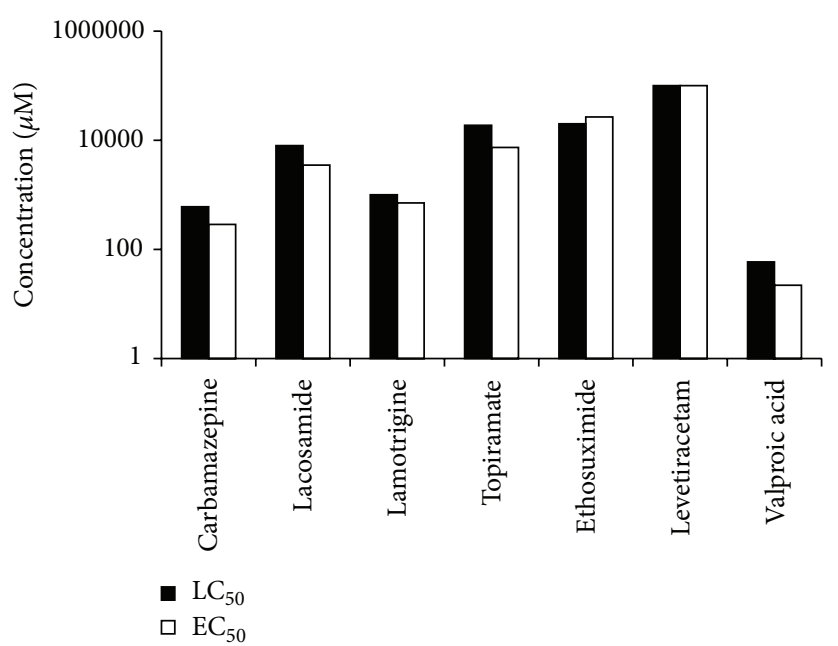

FIGURE 2: Lethal and teratogenic effects of antiepileptic drugs on zebrafish embryos at $72 \mathrm{hpf}$.

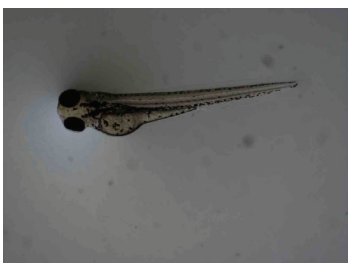

(a)

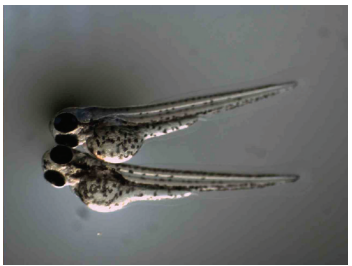

(e)

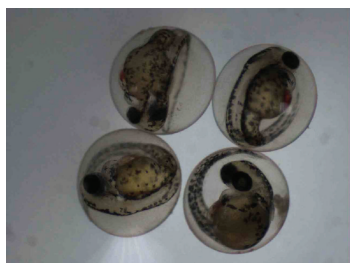

(b)

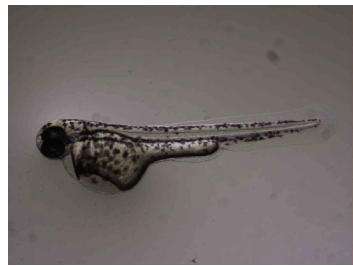

(f)

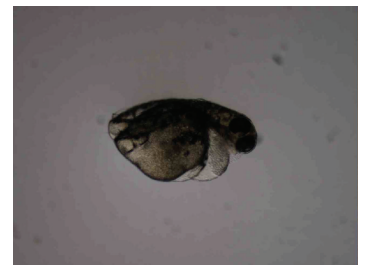

(c)

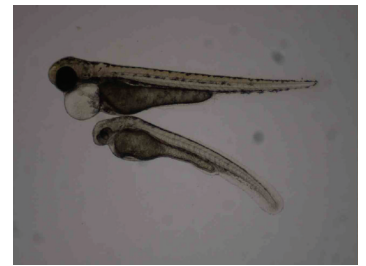

(g)

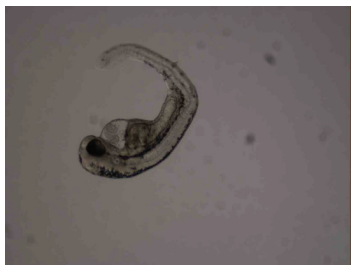

(d)

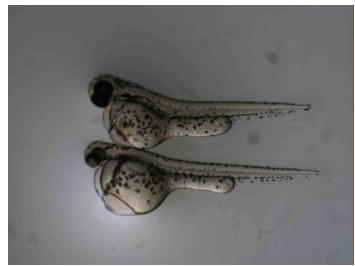

(h)

Figure 3: Zebrafish Embryos at 72 hpf. (a) Normal, $1 \%$ DMSO (×25), (b) 1 mM Carbamazepine ( $\times 30)$, (c) 5 M Valproic acid ( $\times 30)$, (d) $100 \mu$ M Lamotrigine (×35), (e) $1 \mathrm{mM}$ Levetiracetam (×25), (f) $500 \mu \mathrm{M}$ Lacosamide (×30), (g) $10 \mathrm{mM}$ Ethosuximide $(\times 30)$ and (h) $5 \mathrm{mM}$ Topiramate $(\times 25)$.

scoliosis, deformity of yolk, and growth retardation) were evaluated under a microscope at $72 \mathrm{hpf}$ (Figure 3). Data are presented in Table 1.

LCM and LMT induced several kinds of malformations and showed significant difference between dose levels. There was some specific type's induction of malformation according to drugs. The main malformation of VPN was growth retardation, and TPM-induced multimalformation included heart edema, yolk deformity, and scoliosis.

3.3. Comparison of TI and Human Pregnancy Categories. The calculated TI values of the compounds were compared with the US FDA human pregnancy category (Table 2, Figure 2). Three drugs CBM, TPM, and VPN, which were classified as pregnancy category $\mathrm{D}$ by FDA and showed $\mathrm{T} 1$ values greater than 2, while the four drugs, ETX, LMT, LCM, and LVT, which were classified as category $\mathrm{C}$ by FDA, showed a wide range of $\mathrm{T} 1$ values between 0.76 and 2.3.

\section{Discussion}

Standard developmental toxicity studies are generally expensive and time-consuming and combinations of antiepileptic drugs are not tested preclinically; the use of zebrafish has the potential to provide a level of predictivity that is as good as or better than that of these current models [15].

It was reported that zebrafish model was very successful in discriminating between teratogens and nonteratogens, having an $87 \%$ concordance with in vivo mammalian data and both a low false-positive and low false-negative rates of 15 and $11 \%$, respectively [4]. The concordance between zebrafish embryo and mammalian models of developmental toxicity ranges from $64 \%$ to $100 \%$ [9]. The teratogenic potential of compounds can be predicted quantitatively by ranking zebrafish embryos based on a scoring system for phenotypic changes that is conceptually similar to morphological assessments conducted using in vivo embryo-fetal development of mammals [15]. 
TABLE 1: Malformation effects of antiepileptic drugs on zebrafish.

\begin{tabular}{|c|c|c|c|c|c|c|c|c|c|c|c|}
\hline & \multicolumn{3}{|c|}{ Embryo numbers $(n)$} & \multicolumn{8}{|c|}{ Malformation in embryos $(n)$} \\
\hline & Treated & Lethal (\%) & Normal (\%) & Observed & Head & Tail & Heart & Yolk & Scoliosis & $\begin{array}{l}\text { Growth } \\
\text { retardation }\end{array}$ & Teratogenicity \\
\hline \multicolumn{12}{|c|}{ Carbamazepine } \\
\hline $2 \mathrm{mM}$ & 10 & $10(100 \%)$ & - & 0 & - & - & - & - & - & - & - \\
\hline $1 \mathrm{mM}$ & 16 & $12(75 \%)$ & $0(0 \%)$ & 4 & $0(0 \%)$ & $0(0 \%)$ & $0(0 \%)$ & $0(0 \%)$ & $0(0 \%)$ & $4(100 \%)$ & $4(100 \%)$ \\
\hline $0.5 \mathrm{mM}$ & 19 & $10(53 \%)$ & $1(11 \%)$ & 9 & $0(0 \%)$ & $0(0 \%)$ & $4(44 \%)$ & $0(0 \%)$ & $1(11 \%)$ & $6(67 \%)$ & $8(89 \%)$ \\
\hline $0.25 \mathrm{mM}$ & 10 & $2(20 \%)$ & $5(50 \%)$ & 8 & $0(0 \%)$ & $0(0 \%)$ & $2(25 \%)$ & $0(0 \%)$ & $0(0 \%)$ & $1(13 \%)$ & $3(38 \%)$ \\
\hline $0.1 \mathrm{mM}$ & 20 & $7(35 \%)$ & $9(45 \%)$ & 13 & $0(0 \%)$ & $1(8 \%)$ & $3(23 \%)$ & $0(0 \%)$ & $1(8 \%)$ & $2(15 \%)$ & $4(31 \%)$ \\
\hline \multicolumn{12}{|l|}{ Lacosamide } \\
\hline $10 \mathrm{mM}$ & 14 & $7(50 \%)$ & $0(0 \%)$ & 7 & $0(0 \%)$ & $0(0 \%)$ & $0(0 \%)$ & $0(0 \%)$ & $0(0 \%)$ & $7(100 \%)$ & $7(100 \%)^{b}$ \\
\hline $5 \mathrm{mM}$ & 26 & $14(54 \%)$ & $2(8 \%)$ & 12 & $0(0 \%)$ & $2(17 \%)$ & $7(58 \%)$ & $0(0 \%)$ & $4(33 \%)$ & $3(25 \%)$ & $10(83 \%)^{b}$ \\
\hline $2.5 \mathrm{mM}$ & 10 & $3(30 \%)$ & $3(30 \%)$ & 7 & $0(0 \%)$ & $0(0 \%)$ & $1(14 \%)$ & $0(0 \%)$ & $0(0 \%)$ & $3(43 \%)$ & $4(57 \%)^{b}$ \\
\hline $1 \mathrm{mM}$ & 26 & $5(19 \%)$ & $16(62 \%)$ & 21 & $0(0 \%)$ & $1(5 \%)$ & $1(5 \%)$ & $0(0 \%)$ & $1(5 \%)$ & $3(14 \%)$ & $5(24 \%)$ \\
\hline $0.5 \mathrm{mM}$ & 25 & $4(16 \%)$ & $20(80 \%)$ & 21 & $0(0 \%)$ & $1(5 \%)$ & $1(5 \%)$ & $0(0 \%)$ & $1(5 \%)$ & $1(5 \%)$ & $2(10 \%)^{\mathrm{a}}$ \\
\hline \multicolumn{12}{|l|}{ Lamotrigine } \\
\hline $1 \mathrm{mM}$ & 25 & $5(20 \%)$ & $7(28 \%)$ & 20 & $1(5 \%)$ & $1(5 \%)$ & $8(40 \%)$ & $6(30 \%)$ & $7(35 \%)$ & $5(25 \%)$ & $13(65 \%)^{b}$ \\
\hline $0.5 \mathrm{mM}$ & 25 & $6(24 \%)$ & $10(40 \%)$ & 19 & $1(5 \%)$ & $2(11 \%)$ & $4(21 \%)$ & $5(26 \%)$ & $2(11 \%)$ & $2(11 \%)$ & $9(47 \%)$ \\
\hline $0.25 \mathrm{mM}$ & 15 & $2(13 \%)$ & $12(80 \%)$ & 13 & $0(0 \%)$ & $1(8 \%)$ & $0(0 \%)$ & $1(8 \%)$ & $0(0 \%)$ & $0(0 \%)$ & $1(8 \%)$ \\
\hline $0.1 \mathrm{mM}$ & 25 & $9(36 \%)$ & $12(48 \%)$ & 16 & $0(0 \%)$ & $0(0 \%)$ & $3(19 \%)$ & $2(13 \%)$ & $0(0 \%)$ & $0(0 \%)$ & $4(25 \%)$ \\
\hline $0.05 \mathrm{mM}$ & 25 & $9(36 \%)$ & $16(64 \%)$ & 16 & $0(0 \%)$ & $0(0 \%)$ & $0(0 \%)$ & $0(0 \%)$ & $0(0 \%)$ & $0(0 \%)$ & $0(0 \%)^{\mathrm{a}}$ \\
\hline \multicolumn{12}{|l|}{ Topiramate } \\
\hline $50 \mathrm{mM}$ & 10 & $8(80 \%)$ & $0(0 \%)$ & 2 & $0(0 \%)$ & $0(0 \%)$ & $2(100 \%)$ & $2(100 \%)$ & $1(50 \%)$ & $1(50 \%)$ & $2(100 \%)$ \\
\hline $10 \mathrm{mM}$ & 16 & $10(63 \%)$ & $4(25 \%)$ & 6 & $0(0 \%)$ & $0(0 \%)$ & $2(33 \%)$ & $1(17 \%)$ & $2(33 \%)$ & $1(17 \%)$ & $2(33 \%)$ \\
\hline $5 \mathrm{mM}$ & 15 & $3(20 \%)$ & $5(33 \%)$ & 12 & $0(0 \%)$ & $0(0 \%)$ & $4(33 \%)$ & $1(8 \%)$ & $1(8 \%)$ & $3(25 \%)$ & $7(58 \%)$ \\
\hline $1 \mathrm{mM}$ & 14 & $5(36 \%)$ & $3(21 \%)$ & 9 & $0(0 \%)$ & $0(0 \%)$ & $6(67 \%)$ & $2(22 \%)$ & $2(22 \%)$ & $2(22 \%)$ & $6(67 \%)$ \\
\hline $0.5 \mathrm{mM}$ & 15 & $3(20 \%)$ & $9(60 \%)$ & 12 & $0(0 \%)$ & $1(8 \%)$ & $3(25 \%)$ & $0(0 \%)$ & $0(0 \%)$ & $0(0 \%)$ & $3(25 \%)$ \\
\hline \multicolumn{12}{|c|}{ Ethosuximide } \\
\hline $100 \mathrm{mM}$ & 15 & $15(100 \%)$ & - & 0 & - & - & - & - & - & - & - \\
\hline $50 \mathrm{mM}$ & 15 & $9(60 \%)$ & $2(0 \%)$ & 6 & $0(0 \%)$ & $0(0 \%)$ & $0(0 \%)$ & $0(0 \%)$ & $0(0 \%)$ & $4(67 \%)$ & $4(67 \%)$ \\
\hline $10 \mathrm{mM}$ & 15 & $7(47 \%)$ & $1(7 \%)$ & 8 & $0(0 \%)$ & $0(0 \%)$ & 7 (88\%) & $0(0 \%)$ & $0(0 \%)$ & $6(75 \%)$ & 7 (88\%) \\
\hline $5 \mathrm{mM}$ & 15 & $6(40 \%)$ & $9(60 \%)$ & 9 & $0(0 \%)$ & $0(0 \%)$ & $0(0 \%)$ & $0(0 \%)$ & $0(0 \%)$ & $0(0 \%)$ & $0(0 \%)$ \\
\hline $1 \mathrm{mM}$ & 15 & $6(40 \%)$ & $9(60 \%)$ & 9 & $0(0 \%)$ & $0(0 \%)$ & $0(0 \%)$ & $0(0 \%)$ & $0(0 \%)$ & $0(0 \%)$ & $0(0 \%)$ \\
\hline \multicolumn{12}{|c|}{ Levetiracetam } \\
\hline $100 \mathrm{mM}$ & 14 & $6(43 \%)$ & $7(50 \%)$ & 8 & $0(0 \%)$ & $1(13 \%)$ & $0(0 \%)$ & $0(0 \%)$ & $0(0 \%)$ & $1(13 \%)$ & $1(13 \%)$ \\
\hline $50 \mathrm{mM}$ & 15 & $6(40 \%)$ & $9(60 \%)$ & 9 & $0(0 \%)$ & $0(0 \%)$ & $0(0 \%)$ & $0(0 \%)$ & $0(0 \%)$ & $0(0 \%)$ & $0(0 \%)$ \\
\hline $10 \mathrm{mM}$ & 10 & $5(50 \%)$ & $5(50 \%)$ & 5 & $0(0 \%)$ & $0(0 \%)$ & $0(0 \%)$ & $0(0 \%)$ & $0(0 \%)$ & $0(0 \%)$ & $0(0 \%)$ \\
\hline $5 \mathrm{mM}$ & 15 & $6(40 \%)$ & $9(60 \%)$ & 9 & $0(0 \%)$ & $0(0 \%)$ & $0(0 \%)$ & $0(0 \%)$ & $0(0 \%)$ & $0(0 \%)$ & $0(0 \%)$ \\
\hline $1 \mathrm{mM}$ & 15 & $7(47 \%)$ & $7(47 \%)$ & 8 & $0(0 \%)$ & $0(0 \%)$ & $1(13 \%)$ & $0(0 \%)$ & $0(0 \%)$ & $0(0 \%)$ & $1(13 \%)$ \\
\hline \multicolumn{12}{|c|}{ Valproic acid } \\
\hline $100 \mu \mathrm{M}$ & 12 & $12(100 \%)$ & - & 0 & - & - & - & - & - & - & - \\
\hline $50 \mu \mathrm{M}$ & 20 & $3(15 \%)$ & $4(20 \%)$ & 17 & - & - & - & - & - & $13(76 \%)$ & $13(76 \%)^{b}$ \\
\hline $25 \mu \mathrm{M}$ & 20 & $2(10 \%)$ & $1(5 \%)$ & 18 & - & - & - & - & - & 17 (94\%) & $17(94 \%)^{b}$ \\
\hline $12.5 \mu \mathrm{M}$ & 20 & $3(15 \%)$ & $13(65 \%)$ & 17 & - & - & - & - & - & $4(24 \%)$ & $4(24 \%)^{\mathrm{a}}$ \\
\hline $6.25 \mu \mathrm{M}$ & 20 & $3(15 \%)$ & $15(75 \%)$ & 17 & - & - & - & - & $1(5 \%)$ & $2(12 \%)$ & $2(12 \%)^{\mathrm{a}}$ \\
\hline $1 \%$ DMSO & 28 & $2(7 \%)$ & $26(93 \%)$ & 28 & $0(0 \%)$ & $0(0 \%)$ & $1(4 \%)$ & $0(0 \%)$ & $1(4 \%)$ & $0(0 \%)$ & $2(7 \%)$ \\
\hline
\end{tabular}


TABLE 2: Comparison of human pregnancy category and zebrafish TI values.

\begin{tabular}{lcc}
\hline Compound & $\begin{array}{c}\text { Human pregnancy } \\
\text { category by FDA }\end{array}$ & Zebrafish TI \\
\hline Carbamazepine & $\mathrm{D}$ & 2.1 \\
Lacosamide & $\mathrm{C}$ & 2.3 \\
Lamotrigine & $\mathrm{C}$ & $>1.4$ \\
Topiramate & $\mathrm{D}$ & 2.5 \\
Ethosuximide & $\mathrm{C}$ & 0.76 \\
Levetiracetam & $\mathrm{C}$ & $<1$ \\
Valproic acid & $\mathrm{D}$ & 2.68 \\
\hline
\end{tabular}

The teratogenicity of antiepileptic drugs is a well-defined subject. The incidences of major malformations include spina bifida, cleft palate, limb reduction defects, cardiac abnormalities, hypospadias, and gastrointestinal atresia [16]. The exact mechanism by which the AEDs mediate abnormalities in the fetus is uncertain. VPN, which was known as inhibitior of histone deacetylases (HDACs), was well investigated and known as generate malformations such as edema, brain deformities, a shortened and bent tail, and bipartite axiation of the posterior trunk in zebrafish. The effects of zebrafish were similar to those observed in mammals [17].

The effects of teratogenic agents on developing organs are susceptible to the developmental stage at the time of exposure. There are critical periods of susceptibility to organ systems affected by these agents. In rat teratogenic study, test compounds are commonly exposed from implantation to closure of the hard palate during which major organ formation occurs, and in zebrafish, rapid morphogenesis is completed at hatching stage which is a similar stage to rat development [18]. So we observed zebrafish embryos at $72 \mathrm{hrs}$ and collected malformations data in this study.

In this experiment, we tried to discriminate the embryotoxic effect (lethality) and malformation effect (teratogenicity) of 7 AEDs in zebrafish model. The 7 AEDs showed large range of $\mathrm{LC}_{50}$ and $\mathrm{EC}_{50}$. In $\mathrm{VPN}$, the $\mathrm{LC}_{50}$ was $59 \mu \mathrm{M}$, which was the lowest lethal concentration, and the $\mathrm{E}_{50}$ was $22 \mu \mathrm{M}$, whose observed teratogenic effect was mainly growth retardation. The other side, the $\mathrm{LC}_{50}$ of $\mathrm{LVT}$, was over $100 \mathrm{mM}$, and the $\mathrm{EC}_{50}$ of LVT was over $100 \mathrm{mM}$, which did not increase teratogenic effect over lethal dose.

Based on $\mathrm{LC}_{50}$ and $\mathrm{EC}_{50}$ values, a teratogenic index (TI) was calculated. A greater TI value is associated with a toxic agent that produces wide separations between the malformation and lethality dose-response curves. It is possible to have a toxic agent that causes severe malformations but not mortality; conversely, a potentially developmental toxic chemical can be so lethal that malformations are not observed [14]. Three drugs which were classified as pregnancy category D by FDA showed greater than 2 of TI values. But the other four drugs, which were classified as category $\mathrm{C}$ by FDA, showed a wide range of TI values between 0.76 and 2.3.

Although zebrafish have been extensively used as a model in toxicity testing, they were relatively uninformative for understanding the underlying biological complexity and for reducing the uncertainties in predicted outcomes, especially in humans, due to (1) species differences, (2) variability in outcomes, and (3) uncertainties in extrapolating outcomes from the high-dose, short-term animal bioassay exposure regimens to the more common low-dose chronic exposure scenarios experienced in humans.

In our experiments, three compounds classified as pregnancy category D showed TI values greater than 2 . The sensitivity of this study model to humans is $100 \%(3 / 3)$. Zebrafish model could support teratogenic screening model as an alternative model for developmental toxicity studies to predict effects in humans. Zebrafish model was useful screening model for teratogenic potential of antiepilepsy drugs and was in concordance with in vivo mammalian data and human clinical data.

\section{Acknowledgments}

This work was supported by the BioGreen 21 Program of the Rural Development Administration (Grant no. PJ009060) and Bio-Industry Technology Development Program (Grant no. IPET312060-5), Ministry for Food, Agriculture, Forestry and Fisheries, Republic of Korea.

\section{References}

[1] M. J. Eadie, "Antiepileptic drugs as human teratogens," Expert Opinion on Drug Safety, vol. 7, no. 2, pp. 195-209, 2008.

[2] C. L. Harden, "Antiepileptic drug teratogenesis: what are the risks for congenital malformations and adverse cognitive outcomes?" International Review of Neurobiology, vol. 83, pp. 205213, 2008.

[3] T. Tomson and D. Battino, "Teratogenic effects of antiepileptic drugs," Seizure, vol. 17, no. 2, pp. 166-171, 2008.

[4] K. C. Brannen, J. M. Panzica-Kelly, T. L. Danberry, and K. A. Augustine-Rauch, "Development of a zebrafish embryo teratogenicity assay and quantitative prediction model," Birth Defects Research B, vol. 89, no. 1, pp. 66-77, 2010.

[5] D. S. Hill, B. J. Wlodarczyk, A. M. Palacios, and R. H. Finnell, "Teratogenic effects of antiepileptic drugs," Expert Review of Neurotherapeutics, vol. 10, no. 6, pp. 943-959, 2010.

[6] C. Ton, Y. Lin, and C. Willett, "Zebrafish as a model for developmental neurotoxicity testing," Birth Defects Research A, vol. 76, no. 7, pp. 553-567, 2006.

[7] P. McGrath and C. Li, "Zebrafish: a predictive model for assessing drug-induced toxicity," Drug Discovery Today, vol. 13, no. 9-10, pp. 394-401, 2008.

[8] M. Westerfield, The Zebrafish Book: A Guide for the Laboratory Use of Zebrafish (Danio rerio), University of Oregon Press, Eugene, Ore, USA, 5th edition, 2007.

[9] N. S. Sipes, S. Padilla, and T. B. Knudsen, "Zebrafish: as an integrative model for twenty-first century toxicity testing," Birth Defects Research C, vol. 93, no. 3, pp. 256-267, 2011.

[10] M. Meadows, "Pregnancy and the drug dilemma," FDA Consumer Magazine, vol. 35, no. 3, pp. 16-20, 2001.

[11] C. B. Kimmel, W. W. Ballard, S. R. Kimmel, B. Ullmann, and T. F. Schilling, "Stages of embryonic development of the zebrafish," Developmental Dynamics, vol. 203, no. 3, pp. 253-310, 1995.

[12] S. Weigt, N. Huebler, T. Braunbeck, and F. Landenberg, "Zebrafish teratogenicity test with metabolic activation (mDarT): 
effects of phase I activation of acetaminophen on zebrafish Danio rerio embryos," Toxicology, vol. 275, no. 1-3, pp. 36-49, 2010.

[13] S. Weigt, N. Huebler, R. Strecker, T. Braunbeck, and T. H. Broschard, "Zebrafish (Danio rerio) embryos as a model for testing proteratogens," Toxicology, vol. 281, no. 1-3, pp. 25-36, 2011.

[14] M. J. Reimers, A. R. Flockton, and R. L. Tanguay, "Ethanol and acetaldehyde-mediated developmental toxicity in zebrafish," Neurotoxicology and Teratology, vol. 26, no. 6, pp. 769-781, 2004.

[15] R. Chapin, K. A. Rauch, B. Beyer et al., "State of the art in developmental toxicity screening methods and a way forward: a meeting report addressing embryonic stem cells, whole embryo culture, and zebrafish," Birth Defects Research B, vol. 83, no. 4, pp. 446-456, 2008.

[16] L. Etemad, M. Moshiri, and S. A. Moallem, "Epilepsy drugs and effects on fetal development: potential mechanisms," Journal of Research in Medical Sciences, vol. 17, no. 9, pp. 876-881, 2012.

[17] K. Herrmann, "Effects of the anticonvulsant drug valproic acid and related substances on the early development of the zebrafish (Brachydanio rerio)," Toxicology in Vitro, vol. 7, no. 1, pp. 41-54, 1993.

[18] I. W. T. Selderslaghs, A. R. van Rompay, W. D. Coen, and H. E. Witters, "Development of a screening assay to identify teratogenic and embryotoxic chemicals using the zebrafish embryo," Reproductive Toxicology, vol. 28, no. 3, pp. 308-320, 2009. 

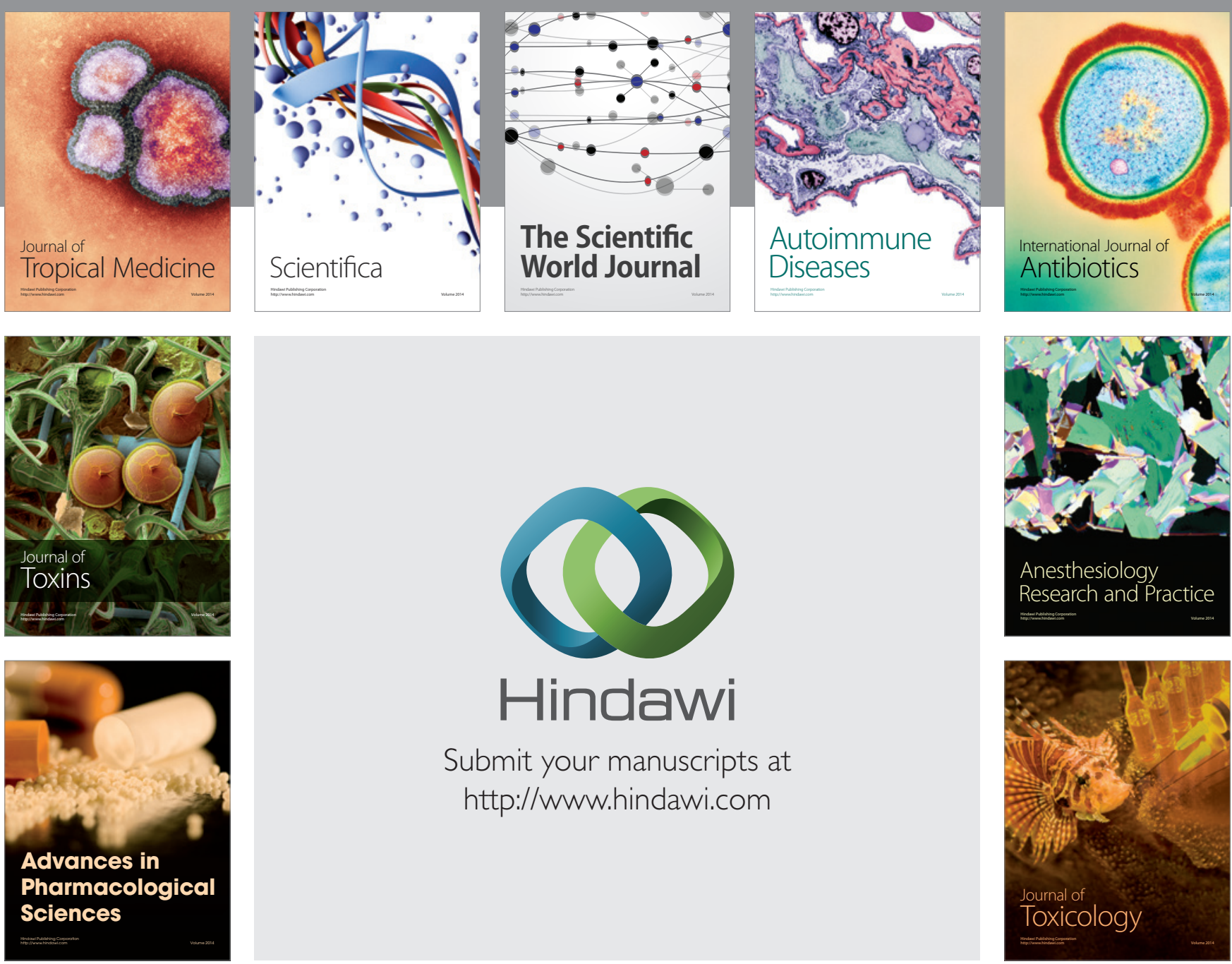

\section{Hindawi}

Submit your manuscripts at

http://www.hindawi.com
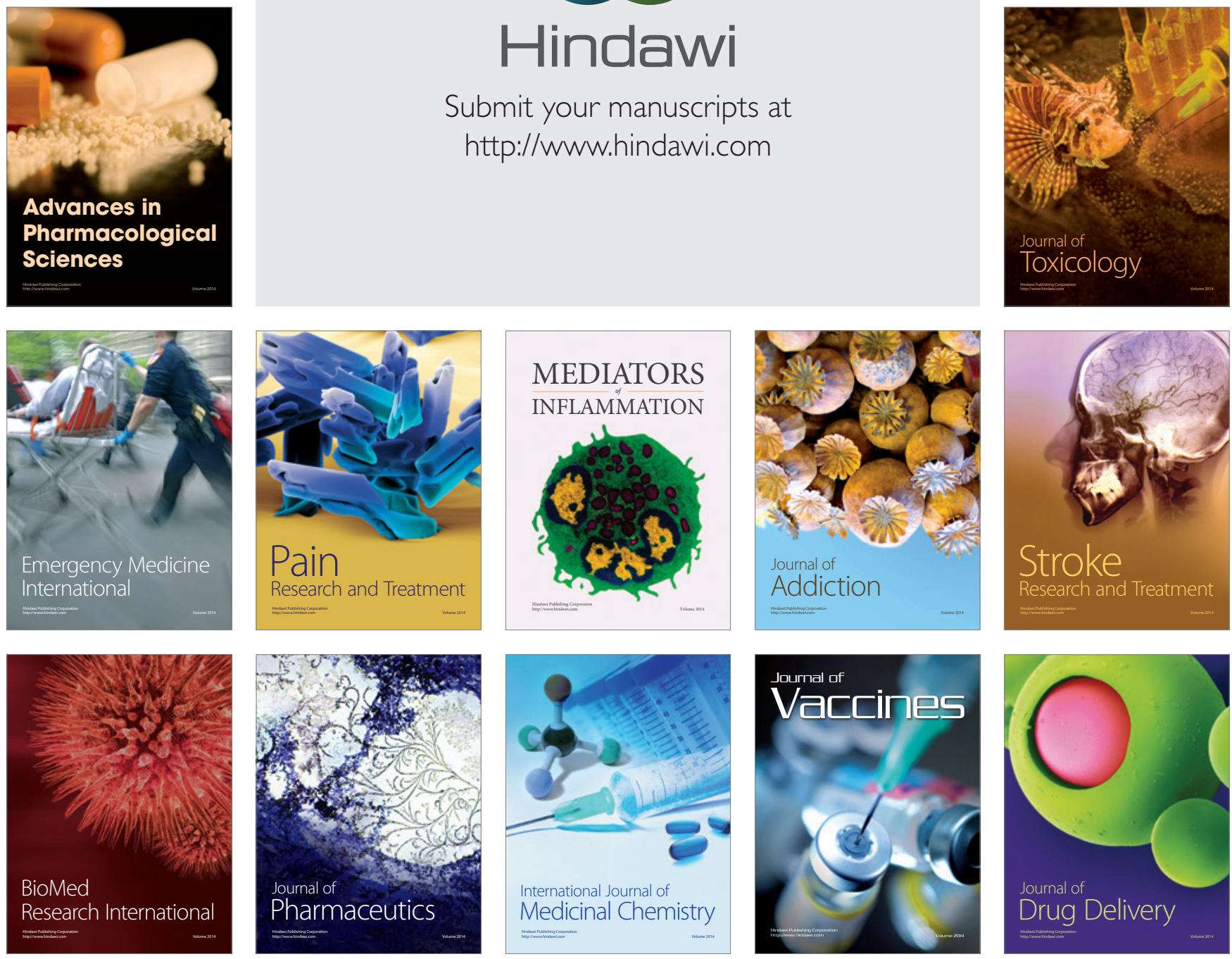